\title{
Minimize the extent and morbidity of axillary dissection for node-positive breast cancer patients: implementation of axillary lymph node dissection based on breast lymphatics level
}

\author{
Qianqian Yuan, Jinxuan Hou, Yukun He, Yiqian Liao, Lewei Zheng and Gaosong Wu* (i)
}

\begin{abstract}
Background: Breast cancer-related lymphedema (BCRL) is associated with extensive axillary dissection. Axillary lymph node dissection (ALND) based on breast lymphatics level (BLL) was proposed to minimize the surgical extent for node-positive breast cancer patients.

Methods: A total of 156 consecutive sentinel lymph node-positive (SLN+) or clinically node-positive (cN+) patients underwent sentinel lymph node biopsy (SLNB) with indocyanine green and methylene blue (MB). The SLNs were injected with $0.1 \mathrm{ml} \mathrm{MB}$ before removal, and a standard ALND was subsequently performed. The nodes adjacent to the blue-stained axillary lymph nodes from the breast (bALNs) were sent for pathological examination separately by resecting serial tissue every $0.5 \mathrm{~cm}$ away from the marginal blue-stained bALNs. Then, a pilot study comparing ALND based on BLL and standard ALND was performed.

Results: BLL were successfully identified in $20 \mathrm{SLN}+(100 \%)$ and $134 \mathrm{cN}+(98.5 \%)$ patients. The median number of BLL was four, ranging from three to six. A horizontal line $1.0 \mathrm{~cm}$ away from the superior blue-stained bALN and a vertical line $1.0 \mathrm{~cm}$ away from the medial blue-stained bALN formed BLL II, III, and IV. All of the additional positive nodes were within $1.0 \mathrm{~cm}$ of the blue-stained bALNs. The minimized axillary dissection should resect upwards from the lowest BLL that contains the first confirmed negative blue-stained bALNs. In the pilot study, no patient developed axillary recurrence.
\end{abstract}

Conclusion: The ALND surgical procedure based on BLL could minimize the surgical extent for pathological nodepositive breast cancer patients and potentially reduce the BCRL rate.

Trial registration: ChiCTR1800014247.

Keywords: Breast cancer, Axillary lymph node dissection, Breast cancer related lymphedema

\footnotetext{
* Correspondence: wugaosong@whu.edu.cn

Department of Thyroid and Breast Surgery, Zhongnan Hospital of Wuhan University, 169 Donghu Road, Wuhan 430071, Hubei, People's Republic of China
}

(c) The Author(s). 2021 Open Access This article is licensed under a Creative Commons Attribution 4.0 International License, which permits use, sharing, adaptation, distribution and reproduction in any medium or format, as long as you give appropriate credit to the original author(s) and the source, provide a link to the Creative Commons licence, and indicate if changes were made. The images or other third party material in this article are included in the article's Creative Commons licence, unless indicated otherwise in a credit line to the material. If material is not included in the article's Creative Commons licence and your intended use is not permitted by statutory regulation or exceeds the permitted use, you will need to obtain permission directly from the copyright holder. To view a copy of this licence, visit http://creativecommons.org/licenses/by/4.0/ The Creative Commons Public Domain Dedication waiver (http://creativecommons.org/publicdomain/zero/1.0/) applies to the data made available in this article, unless otherwise stated in a credit line to the data. 


\section{Background}

The advent of sentinel lymph node biopsy (SLNB) has revolutionized the surgical management of axilla in breast cancer patients [1]. Complete axillary lymph node dissection (ALND) has been gradually replaced for select patients, owing to the substantial morbidity such as debilitating breast cancer-related lymphedema (BCRL), shoulder dysfunction, paresthesia and discomfort [2]. Damage to the lymphatic drainage system in the axilla by multidisciplinary treatment contributes to the occurrence of BCRL [3]. Notably, extensive axillary dissection is related to a high occurrence rate of BCRL. A total of $77.8 \%$ of the patients who underwent ALND had fewer than three additional involved nodes in the ACOSOG Z0011 trial [4]. Thus, the complete removal all axillary lymph nodes with ALND for those patients might be over-treatment. For patients who were not eligible for the ACOSOG Z0011 study criteria, especially clinically node-positive breast cancer patients, a method for deescalating the surgical area of ALND would significantly reduce the $B C R L$ rate.

The lymphatic system of the breast forms an extensive and complex network of periductal and perilobular vessels that drain principally to the axillary nodes [5]. Breast tumors commonly invade local structures and spread in a progressive and sequential manner to regional nodes, and the lymphatic vessels provide anatomical continuity for this process by acting as a link between the primary tumor and the regional nodes. Hence, the sentinel lymph node (SLN) hypothesis presupposes an orderly spread of cancer cells from the primary tumor to the first draining node, which has prevented clinically node-negative breast cancer patients from undergoing extensive axillary dissection [6].

On the theoretical basis of SLNB, for node-positive breast cancer patients, metastasis from breast tumors does not involve the axillary lymph nodes from the breast (bALNs) as a unit but instead progresses from the primary tumor to the first draining nodes, then second and third echelon nodes $[7,8]$. Therefore, our institution implemented the approach of ALND based on the lymphatic drainage from the breast in order to limit surgical resection in the axilla and reduce the morbidity of ALND. The primary aim was to accurately determine the extent of every breast lymphatics level (BLL). The secondary aim was to determine the skip metastasis rate to demonstrate the de-escalation feasibility of ALND based on lymphatic drainage from the breast.

\section{Methods}

\section{Study cohort}

The prospective observational study enrolled consecutive breast cancer patients from November 2017 to March 2018 in a single tertiary referral academic medical center, Zhongnan Hospital of Wuhan University. Women patients meeting the following criteria and undergoing ALND were included in the study: i) patients with clinically node-positive breast cancer, defined as positive on preoperative axillary palpation, ultrasound examinations or CT scans with contrast; ii) patients who underwent mastectomy with a positive SLN; iii) patients who underwent breast-conserving surgery (BCS) for more than two positive SLNs; iv) patients who did not receive neoadjuvant chemotherapy (NCT); v) patients with no previous history of breast cancer. Those who had benign tumors and those who did not meet the above criteria were excluded from the study. The Medical Ethics Committee of Zhongnan Hospital of Wuhan University approved the routine use of the SLNB procedure for all patients. The trial was registered at the Chinese Clinical Trial Registry (ChiCTR1800014247). All patients provided written informed consent regarding SLNB and ALND.

\section{Dual tracers for SLNB}

All patients underwent quadrantectomy or mastectomy immediately followed by SLNB. Radioactive tracers were unavailable in China. Instead, dual tracers, $1 \mathrm{ml}(1 \mathrm{mg})$ indocyanine green (ICG) (H20045514; Weicai Pharmaceutical Corporation, Liaoning, China) and $0.5 \mathrm{ml}$ (5 mg) methylene blue (MB) (H32024827; Jichuan Pharmaceutical Corporation, Taixin, China) were administered for SLNB [9].

\section{Surgical techniques}

Before removal, all the identified SLNs were routinely and meticulously injected with $0.1-0.2 \mathrm{ml} \mathrm{MB}$ using a 1cc syringe with a 32-gauge needle, which was called 'staged tracing' (Supplement 1. MP4). The careful reinjection of $\mathrm{MB}$ was a key procedure to not artifactually alter the lymphatic drainage patterns. $M B$ could then flow from the SLNs along several ascending lymphatic channels towards the subclavian lymph nodes. Then, the blue-stained lymphatic channels were mapped by bluntly dissecting along the lymphatic drainage channels from the breast to the axilla. After identifying the efferent and echelon nodes, the SLNs were ready to be harvested and sent for immediate frozen sectioning (FS). Once the SLNs were confirmed positive in patients who underwent mastectomy, ALND was performed with complete resection of at least Berg's levels I and II; the resection of Berg's level III was performed only in patients with gross disease in Berg's level II and/or III. When patients with BCS had more than two positive SLNs, ALND was subsequently performed.

After complete ALND, the removed specimen was dissected carefully ex vivo (Fig. 1). The lymph nodes adjacent to the blue-stained bALNs were defined as 


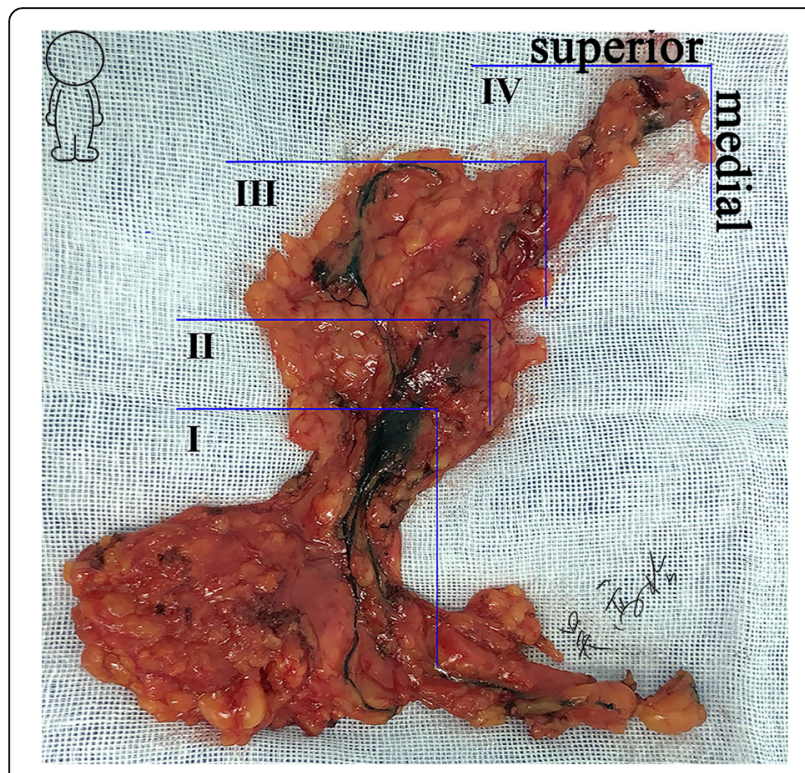

Fig. 1 Breast lymphatics level in this study (ex vivo, right axilla)

nonstained bALNs (Fig. 2). A horizontal line along the superior blue-stained bALN and a vertical line along the medial blue-stained bALN formed a lower outer quadrant (LOQ) zone in the axilla, which was defined as the BLL (Fig. 2). The blue-stained lymphatic channels of the breast could converge towards a group of three to five lymph nodes, namely, SLNs, at BLL I. From these nodes,

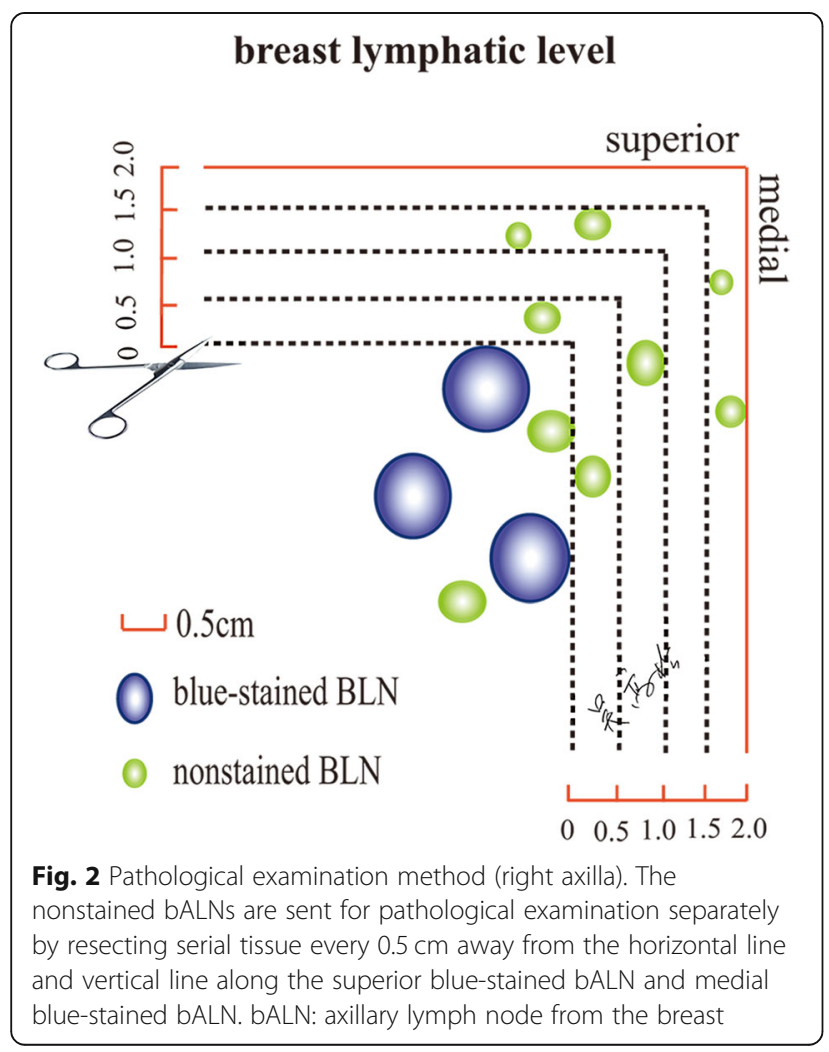

there was a predictable passage of efferent lymphatics towards nodes at BLL II and, in turn, nodes at BLL III and IV (Fig. 1). With efferent lymphatics, each BLL could be distinguished. The skip metastasis was defined as macrometastatic nodes that were found at a further site while the lymph nodes nearer to the primary breast tumor were negative, as it was assumed that nodal involvement occurred in a progressive manner.

\section{Pathological examination}

The removed SLNs were sent for immediate FS during the operation. After the operation, the nonstained bALNs were sent for pathological examination separately by resecting serial tissue every $0.5 \mathrm{~cm}$ away from the horizontal line and vertical line along the marginal blue-stained bALN (Fig. 2) [10]. All the retrieved bALNs were processed for routine hematoxylin and eosin staining for histology and immunohistochemically. The diameter, estrogen receptor status, progesterone receptor status and human epidermal growth factor receptor-2 status of the primary tumor were also assessed as part of a routine pathology examination.

\section{Comparing ALND based on BLL with standard ALND}

Patients with clinical $\mathrm{T}_{1-3}$ and node-positive axilla were eligible and randomized to ALND based on BLL and standard ALND. Descriptive statistics were used to describe the results of the pilot prospective study. The primary objective of the pilot phase of the randomized controlled trial was to demonstrate feasibility of the procedure of ALND based on BLL trial design, and to determine if axillary recurrence rates for patients randomized to ALND based on BLL are equivalent to axillary recurrence rates for patients randomized to standard ALND.

\section{Statistical analysis}

The demographic characteristics, tumor sizes, and number of lymph nodes in each BLL were collected. Continuous variables between groups were compared using a nonparametric test. Chi-squared tests or Fisher's exact tests were run to compare the positive rate between the two groups. Two-sided $p$ values $<0.05$ were considered statistically significant. All statistical analyses were performed using SPSS17.0 for Windows (SPSS, Inc., Chicago, IL).

\section{Results}

\section{Patients}

A total of 190 patients underwent SLNB with dual tracers. Of these patients, 136 had node-positive axilla, and 54 patients had node-negative axilla. Two patients who failed to have any SLNs identified, 26 patients who underwent mastectomy with negative SLNs, and 6 patients who underwent BCS and planned whole-breast 
irradiation with fewer than 3 macrometastastic SLNs were excluded. Of the 26 patients with negative SLN identified by FS, two (7.7\%) false negative cases with one lymph node were found to have macrometastatic $(2 \mathrm{~mm})$ disease after the postoperative H\&E examination. A total of 156 patients were entered into the study, and $78.8 \%$ of the patients underwent mastectomy. Systemic therapy was recommended according to the clinical guidelines. The baseline characteristics of the SLN+ and $\mathrm{cN}+$ groups are shown in Table 1. Compared with the SLN+ group, the $\mathrm{cN}+$ group displayed larger tumors $(p<$ $0.001)$. All of the patients underwent staged tracing to reveal the subsequent efferent and echelon bALNs, and MB could flow from the SLNs along several ascending lymphatic channels towards the subclavian lymph nodes. Twenty (100\%) SLN+ patients and 134 (98.5\%) cN+ patients successfully had the breast lymphatic vessels and bALNs identified (Fig. 1).

Supraclavicular and infraclavicular radiotherapy was performed for patients with one or more positive lymph nodes. Chest wall radiotherapy was performed for patients who underwent BCS. 20 (100\%) SLN+ patients and $121(89.0 \%) \mathrm{cN}+$ patients underwent radiotherapy. The dose was 25 fractions of $2 \mathrm{~Gy}$.

\section{Breast lymphatics level}

The median number of BLL that could be classified was four, ranging from three to six (Fig. 1). The mean number of lymph nodes in each BLL is presented in Table 2. The skip metastasis rate of different serial distances from the marginal blue-stained bALNs is described in Table 3. While the cancer cells were not found in the blue-stained bALNs, the additional skip macrometastatic lymph nodes were located within $1.0 \mathrm{~cm}$ distance away from the bluestained bALNs. No additional macrometastatic nodes were identified beyond $1.0 \mathrm{~cm}$ away from the marginal bALNs (Table 3). Therefore, a horizontal line $1.0 \mathrm{~cm}$ away from the superior blue-stained bALN and a vertical line $1.0 \mathrm{~cm}$ away from the medial blue-stained bALN formed a LOQ zone in the axilla, which was defined as BLL II, III, and IV (Fig. 3b). The skip metastasis rate of SLN was 9.6\%, which was higher than the subsequent lymph nodes (Table 3). And no additional macrometastatic nodes were identified beyond $1.5 \mathrm{~cm}$ away from the marginal blue-stained SLNs. Thus, a horizontal line $1.5 \mathrm{~cm}$ away from the superior blue-stained SLN and a vertical line $1.5 \mathrm{~cm}$ away from the medial blue-stained SLN formed a LOQ zone in the axilla, which was defined as BLL I [10].

The location of the BLL was dynamic and inconsistent. The BLL I was lateral and inferior to the pectoralis minor muscle; BLL II was either located lateral and inferior to the pectoralis minor, behind the pectoralis minor, or both. In addition, BLL III was located behind the pectoralis minor, medial and superior the pectoralis minor, or both; only later BLL IV lymph nodes were medial and superior to the muscle.

\section{Pathology}

Ninety-seven (62.2\%) of the 156 patients harbored fewer than four macrometastatic lymph nodes. $\mathrm{cN}+$ patients were more prone to have gross metastasis than SLN+ patients $(p<0.001)$. The distribution of the positive nodes in the BLL between the SLN+ and $\mathrm{cN}+$ groups was compared and can be seen in Table 4 . The cancer cells of $85.8 \%(121 / 141)$ of the patients with node-positive axilla metastasized from the primary tumor to BLL II.

\section{Outcome of the pilot study}

The surgical procedure of ALND based on BLL was performed as follows: (1) Perform SLNB with $0.5 \mathrm{ml} \mathrm{MB}$ (Fig. 3a). (2) Routinely and meticulously inject all the identified SLNs with $0.1-0.2 \mathrm{ml}$ of $\mathrm{MB}$ before removal (Fig. 3b). (3) After the echelon nodes were identified with injection of $\mathrm{MB}$, SLNs would be harvested and pathologically examined by immediate FS (Fig. 3c). (4) If SLNs were positive, the blue-stained bALNs in BLL 2nd would be removed and sent for immediate FS (Fig. 3d). (5) If the blue-stained bALNs in BLL 2nd were confirmed negative, the tissues in BLL 2nd were resected 'en bloc' (Fig. 3e); (6) If the blue-stained bALNs in BLL 2nd were macrometastic, the blue-stained bALNs in lymphatic level 3rd would be removed and sent for immediate FS (Fig. 3f). Finally, the limited axillary dissection would be performed upwards from the lowest BLL that contained the first confirmed negative blue-stained bALNs, and the tissues in the matched LOQ zone would be resected en bloc.

Twenty-seven patients were enrolled in the pilot study from March 2018 to July 2018. Fourteen participants were randomized to ALND based on BLL (study group), 13 subjects were randomized to standard ALND (controlled group), and two patients withdrew from the trial. Thirteen patients from study group, and 11 patients from controlled group completed the study interventions and are included in the analysis. The median age in study group was 48.0 (range 39-62 years) and the median age in controlled group was 46.0 (range $31-61$ ). The two groups are well-matched in terms of patient age, tumor size, biomarker profile and length of followup (Table 5). In the ALND based on BLL group, one (1.3\%) false negative case with two lymph nodes were found to have micrometastatic $(1 \mathrm{~mm})$ disease after the postoperative H\&E examination. After a median follow-up of 24 months (range 22-25 months), no axillary recurrence and distant 
Table 1 Characteristics of 156 breast cancer patients

\begin{tabular}{|c|c|c|c|}
\hline & $\begin{array}{l}\mathrm{SLN}+ \\
N=20\end{array}$ & $\begin{array}{l}\mathrm{cN}+ \\
N=136\end{array}$ & $p$ value \\
\hline Age (Mean) & 49.45 & 51.1 & $0.627^{a}$ \\
\hline Tumor size, №. (\%) & & & $<0.001^{\mathrm{a}}$ \\
\hline$<1 \mathrm{~cm}$ & $4(20.0)$ & $6(4.4)$ & \\
\hline $1-2 \mathrm{~cm}$ & $14(70.0)$ & $38(27.9)$ & \\
\hline $2-3 \mathrm{~cm}$ & $2(10.0)$ & $59(43.4)$ & \\
\hline$>3 \mathrm{~cm}$ & 0 & $33(24.3)$ & \\
\hline pN stage, No. (\%) & & & $0.03^{\mathrm{a}}$ \\
\hline NO & 0 & $15(11.0)$ & \\
\hline $\mathrm{N} 1$ & $15(75.0)$ & $67(49.3)$ & \\
\hline N2 & $5(25.0)$ & $45(33.1)$ & \\
\hline N3 & 0 & $9(6.6)$ & \\
\hline Surgery, No. (\%) & & & $<0.001^{\mathrm{b}}$ \\
\hline Mastectomy (a SLN) & $9(45.0)$ & $114(83.8)$ & \\
\hline BCS ( $\geq 3$ positive SLNs) & $11(55.0)$ & $22(16.2)$ & \\
\hline Histological type (\%) & & & $0.882^{\mathrm{a}}$ \\
\hline ER/PR+, HER2- & $12(60.0)$ & $79(58.1)$ & \\
\hline ER/PR+, HER2+ & $2(10.0)$ & $11(8.1)$ & \\
\hline ER-, PR-, HER2+ & $3(15.0)$ & $22(16.2)$ & \\
\hline ER-, PR-, HER2- & $3(15.0)$ & $24(17.6)$ & \\
\hline Ki-67, No. (\%) & & & $0.699^{\mathrm{a}}$ \\
\hline$\geq 14 \%$ & $11(55.0)$ & 81 (59.6) & \\
\hline$<14 \%$ & $9(45.0)$ & $55(40.4)$ & \\
\hline Lymphovascular invasion, No. (\%) & $11(55.0)$ & $83(61.0)$ & $0.607^{\mathrm{a}}$ \\
\hline Chemotherapy, №. (\%) & & & $0.076^{\mathrm{a}}$ \\
\hline Yes & $16(80.0)$ & $292(66.7)$ & \\
\hline No & $4(20.0)$ & $74(33.8)$ & \\
\hline Hormonal therapy, No. (\%) & & & $0.903^{\mathrm{a}}$ \\
\hline Yes & $14(70.0)$ & $97(71.3)$ & \\
\hline No & $6(30.0)$ & $39(28.7)$ & \\
\hline Anti-HER2 therapy, No. (\%) & & & 1.000 \\
\hline Yes & $5(25.0)$ & $33(24.3)$ & \\
\hline No & $15(75.0)$ & $103(75.7)$ & \\
\hline Radiotherapy, No. (\%) & & & $0.248^{\mathrm{a}}$ \\
\hline Yes & $20(100.0)$ & $121(89.0)$ & \\
\hline No & $0(0)$ & $15(11.0)$ & \\
\hline Identification rate of breast lymphatics level, No. (\%) & $20(100)$ & $134(98.5)$ & 1.000 \\
\hline Staged tracing ${ }^{c}$, No. (\%) & $17(85.0)$ & $91(66.9)$ & $0.102^{b}$ \\
\hline
\end{tabular}

SLN+ positive sentinel lymph node, $C N+$ clinically node-positive axilla, $B C S$ breast-conserving surgery

${ }^{a}$ Nonparametric test; ${ }^{b}$ Chi-squire test

'Staged tracing: injecting $0.1 \mathrm{ml}$ blue dye into SLNs

metastasis event was observed in the ALND based on BLL group and standard ALND group. One (7.7\%) patients in the ALND based on BLL group and two (18.2\%) patients in the standard group occurred arm lymphedema (Table 5).

\section{Discussion}

In this prospective observational study, ALND based on BLL attempted to resect potentially metastatic tissues level by level to minimize the extent and morbidity of ALND. Staged tracing (injection of $0.1 \mathrm{ml} \mathrm{MB}$ into the 
Table 2 Number of nodes obtained, blue-stained bALNs, and positive nodes for each breast lymphatics level

\begin{tabular}{|c|c|c|c|}
\hline Breast lymphatics level $^{a}$ & No. of nodes obtained ${ }^{b}$ & No. of blue-stained bALNs & No. positive nodes \\
\hline Level I & $5.0(3-8)$ & $3.5(3-5)$ & $3.0(0-8)$ \\
\hline Level II & $6.5(4-8)$ & $5.5(3-7)$ & $1.0(0-8)$ \\
\hline Level III & $5.5(4-8)$ & $5.0(2-7)$ & $0.8(0-8)$ \\
\hline Level IV & $3.0(2-5)$ & $2.0(1-3)$ & $0.3(0-2)$ \\
\hline Total & $19.5(13-30)$ & $16.5(9-20)$ & $5.1(0-20)$ \\
\hline
\end{tabular}

${ }^{a}$ Median breast lymphatics level was four, level $\mathrm{V}$ and level VI were not listed

${ }^{b}$ No. of nodes obtained: blue-stained axillary lymph nodes from the breast (bALNs) plus non-stained bALNs, mean (mix- max)

SLNs) was utilized to reveal the breast lymphatic system in the axilla basin. The median number of BLL was four, ranging from three to six. A horizontal line $1.0 \mathrm{~cm}$ away from the superior blue-stained bALN and a vertical line $1.0 \mathrm{~cm}$ away from the medial blue-stained bALN formed BLL II, III, IV. The skip metastasis rate was zero when an en bloc resection was performed upwards towards the BLL that contained the first confirmed negative blue-stained bALN. As described in a previous study, a horizontal line $1.5 \mathrm{~cm}$ away from the superior bluestained bALN and a vertical line $1.5 \mathrm{~cm}$ away from the medial blue-stained bALN formed BLL I, which was proposed to be removed en bloc in breast cancer patients with negative SLN to reduce the number of false-negative events from SLNB [10]. In the present study, through resecting the lymph nodes level by level for breast cancer patients with node-positive axilla, the surgical approach of ALND based on BLL was valuable in reducing the BCRL rate without reducing cancer control.

Depending on the various criteria of BCRL and the extent of axillary dissection, a pooled estimation of the arm lymphedema rate is $16.6 \%$ (95\% CI 13.6-20.2) [11]. The risk factors of BCRL can be affected by two aspects: demographic and lifestyle [11], and breast cancer-related variables, including radiotherapy to the axilla, number of nodes involved and removed, and taxane-based chemotherapy. In addition, a hypothesis was proposed that the transection of lymphatic vessels that drain the arm during their course through the axilla during complete ALND was associated with BCRL [12]. Thompson et al.

Table 3 Skip metastasis rate of different serial distances from marginal blue-stained bALNs

\begin{tabular}{llllll}
\hline $\begin{array}{l}\text { Breast } \\
\text { lymphatics } \\
\text { level }\end{array}$ & \multicolumn{5}{l}{ Skip metastasis rate (No.) } \\
\cline { 2 - 6 } $\mathbf{0 . 5} \mathbf{~ c m}^{\mathbf{a}}$ & $\mathbf{0 . 5} \mathbf{~ c m}$ & $\mathbf{1 . 0} \mathbf{~ c m}$ & $\mathbf{1 . 5} \mathbf{~ c m}$ & $\mathbf{2 . 0} \mathbf{~ c m}$ \\
\hline level I & $9.6 \%(15)$ & $7.7 \%(12)$ & $3.8 \%(6)$ & 0 & 0 \\
level II & $6.5 \%(10)$ & $3.9 \%(6)$ & 0 & 0 & 0 \\
level III & $4.5 \%(7)$ & $1.3 \%(2)$ & 0 & 0 & 0 \\
level IV & $3.9 \%(6)$ & $0.6 \%(1)$ & 0 & 0 & 0 \\
\hline
\end{tabular}

${ }^{\mathrm{a}}$ Distance from visualized bALNs. bALN: breast lymph node
[13] and Nos et al. [14]. have previously described a new technique, axillary reverse mapping (ARM), to identify and preserve arm lymph nodes, which reduced the number of arm lymphedema events [15]. A refined ARM technique was proposed in our institution to identify the arm lymphatic system and eliminate postoperative arm lymphedema [16, 17]. An intact pathway for lymphatic arm drainage is adjacent to the axillary vein and is usually located above the second intercostobrachial nerve. Hence, a horizontal line in this study was designed as the upper landmark during ALND surgery based on BLL to protect the arm lymphatic system. In addition, according to the direction of the lymphatic drainage, the medial and superior blue-stained bALNs were selected as the landmark.

Over the past few years, axillary management has changed greatly [18]. With effective multidisciplinary treatment, the theory of breast cancer surgery leans towards "less is more" [19]. After the publication of the ACOSOG-Z0011 and AMAROS trials, varieties of patterns of care for axillary surgery were present [20], particularly for $\mathrm{CT}_{1-2} \mathrm{~N}_{0} \mathrm{M}_{0}$ patients with positive SLNs, which aimed to decrease the treatment-related morbidity without reducing cancer control. For breast cancer patients with $\mathrm{cN}+$ axilla, NCT was often performed and targeted axillary dissection was done to identify the patients who might not require ALND [21]. The omission of complete ALND in these studies was associated with much lower rates of lymphedema. However, clinically node-positive patients who undergo ALND and patients who are not eligible based on the Z0011 criteria also need de-escalate surgical areas for ALND. Approximately $25 \%$ of the patients who undergo SLNB have positive nodes, and these patients undergo ALND and remain at risk for arm lymphedema [22].

As is well-known, metastasis from breast cancer does not involve the breast regional lymph nodes as a unit but rather progresses from the primary tumor to the first-line draining nodes and, in turn, sequentially to the second and third echelon nodes [23]. Based on the biological and anatomical rationale of SLNB, the approach of ALND based on BLL was proposed in our institution to balance the demand of preventing axillary recurrence 


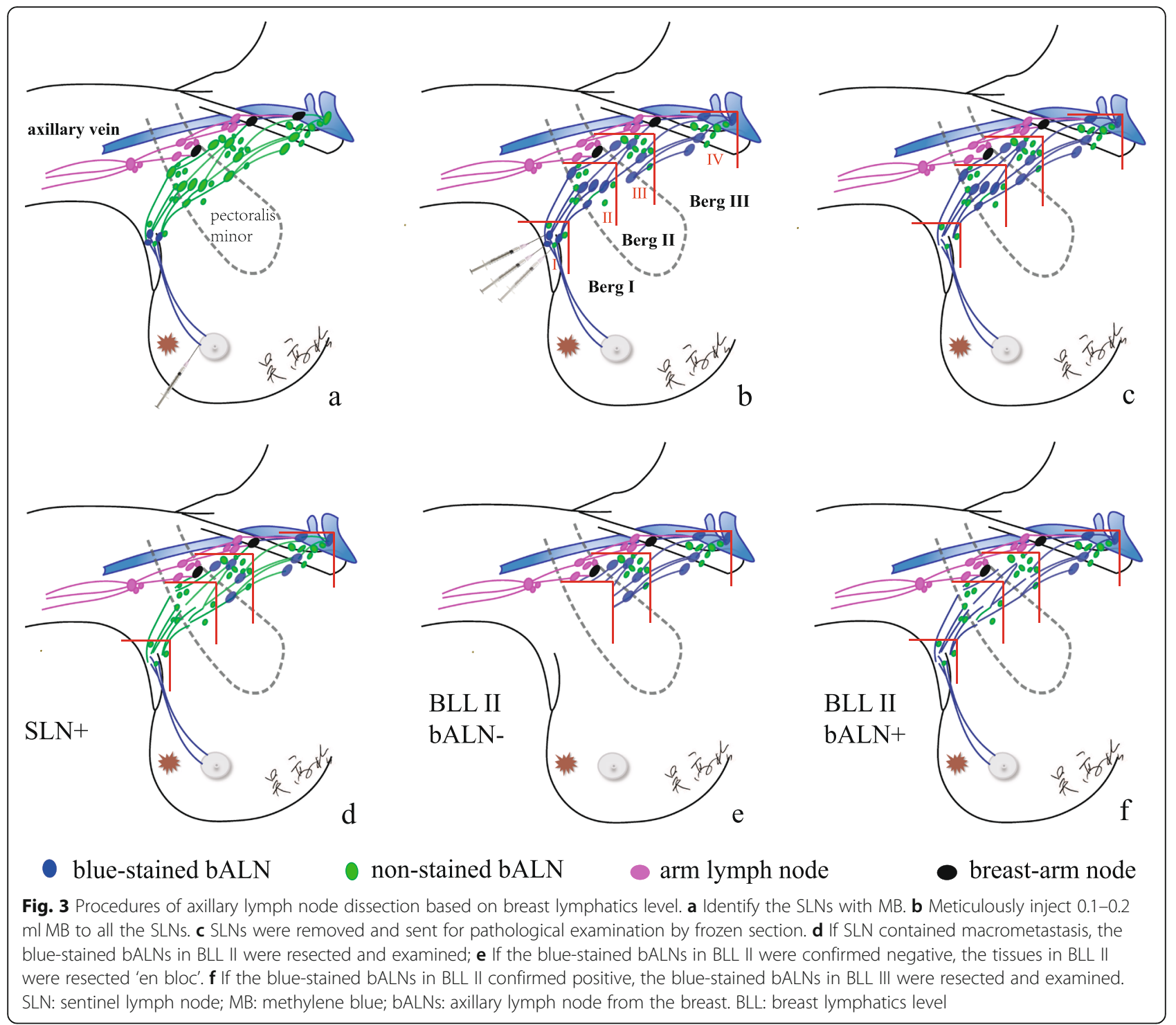

and the wish of avoiding treatment-related morbidity, particularly arm lymphedema. Classifying the bALNs according to lymphatic drainage is a feasible and dynamic way to limit axillary surgical dissection. In the present study, to dispel skip metastasis, nonstained bALNs were sent for pathological examination separately by resecting serial tissue every $0.5 \mathrm{~cm}$ away from the horizontal line

Table 4 The distribution of the positive nodes in the breast lymphatics level between the two groups

\begin{tabular}{llll}
\hline Breast lymphatics level, No. (\%) & $\begin{array}{l}\text { SLN+ } \\
(\boldsymbol{n}=\mathbf{2 0})\end{array}$ & $\begin{array}{l}\mathbf{c N}+ \\
(\boldsymbol{n}=\mathbf{1 2 1})\end{array}$ & $\begin{array}{l}\text { Total } \\
(\boldsymbol{n}=\mathbf{1 4 1})\end{array}$ \\
\hline level I & $15(75)$ & $67(55.4)$ & $82(58.1)$ \\
level II & $4(20)$ & $35(28.9)$ & $39(27.7)$ \\
level III & $1(5)$ & $14(11.6)$ & $15(10.6)$ \\
level IV & 0 & $5(4.1)$ & $5(3.5)$ \\
\hline
\end{tabular}

SLN+ positive sentinel lymph node, $C N+$ clinically node-positive axilla and vertical line along the marginal blue-stained bALN (Fig. 2). In cases of skip metastasis, additional involved nodes were found within the area $1.0 \mathrm{~cm}$ away from the marginal blue-stained bALNs (Table 3). Therefore, a horizontal line $1.0 \mathrm{~cm}$ away from the superior bluestained bALN and a vertical line $1.0 \mathrm{~cm}$ away from the medial blue-stained bALN formed the BLL II, III, IV. The skip metastasis rate was zero when en bloc resection was performed upwards towards the BLL that contained the first confirmed negative blue-stained bALN (Fig. 3f), which could limit the extent of axillary dissection and reduce the number of BCRL events.

Potential limitations existed in the present study. Considering that NCT could influence the structure of the breast lymphatics and lead to an incomplete lymphatic pathway, patients who underwent NCT were excluded from the study. The pilot phase of the randomized 
Table 5 Baseline characteristics of the two group participants in the pilot study

\begin{tabular}{|c|c|c|c|}
\hline & $\begin{array}{l}\text { ALND based on BLL } \\
N=13\end{array}$ & $\begin{array}{l}\text { Standard ALND } \\
N=11\end{array}$ & $p$ value \\
\hline Patients, No. (\%) & & & 0.478 \\
\hline SLN+ & $4(30.8)$ & $2(18.2)$ & \\
\hline $\mathrm{cN}+$ & $9(69.2)$ & $9(81.8)$ & \\
\hline Age, Median (min, max) & $48.0(39,62)$ & $46.0(31,61)$ & $0.239 \mathrm{~b}$ \\
\hline BMI, Mean (min, max) & $24.1(18.4,28.4)$ & $25.4(19.1,28.3)$ & $0.364 b$ \\
\hline No. of nodes removed, mean (min, max) & $8.0(7,17)$ & $11.0(10,18)$ & $<0.001 \mathrm{~b}$ \\
\hline Tumor size, $\mathrm{n}(\%)$ & & & $0.886^{\mathrm{a}}$ \\
\hline $\mathrm{T} 1 \mathrm{~b} / \mathrm{a} / \mathrm{mi}$ & $1(7.7)$ & $1(9.1)$ & \\
\hline T1c & $4(19.2)$ & $3(27.3)$ & \\
\hline $\mathrm{T} 2$ & $7(53.8)$ & $5(45.5)$ & \\
\hline T3 & $1(7.7)$ & $2(18.2)$ & \\
\hline Pathological nodal status, No. (\%) & & & $0.531^{\mathrm{a}}$ \\
\hline$N_{0}$ & 0 & 0 & \\
\hline $\mathrm{N}_{1}$ & $7(53.8)$ & $5(45.5)$ & \\
\hline $\mathrm{N}_{2}$ & $6(46.2)$ & $5(45.5)$ & \\
\hline $\mathrm{N}_{3}$ & 0 & $1(9.1)$ & \\
\hline Tumor grade, No. (\%) & & & $0.850^{\mathrm{a}}$ \\
\hline । & $2(15.4)$ & $2(18.2)$ & \\
\hline II a & $4(30.8)$ & $3(27.3)$ & \\
\hline$\| \mathrm{b}$ & $3(23.1)$ & $4(36.4)$ & \\
\hline III & $4(30.8)$ & $2(18.2)$ & \\
\hline Tumor subtype, No. (\%) & & & $0.967^{\mathrm{a}}$ \\
\hline ER/PR+, HER2- & $6(46.2)$ & $5(45.5)$ & \\
\hline ER/PR+, HER2+ & $2(15.4)$ & $2(18.2)$ & \\
\hline ER-, PR-, HER2+ & $3(23.1)$ & $3(27.3)$ & \\
\hline ER-, PR-, HER2- & $2(15.4)$ & $1(9.1)$ & \\
\hline Ki-67, No. (\%) & & & $0.973^{\mathrm{a}}$ \\
\hline$\geq 14 \%$ & $7(53.8)$ & $6(54.5)$ & \\
\hline$<14 \%$ & $6(46.2)$ & $5(45.5)$ & \\
\hline Lymphovascular invasion, №. (\%) & $8(61.5)$ & 7 (63.6) & 1.000 \\
\hline Chemotherapy, №. (\%) & & & $0.834^{\mathrm{a}}$ \\
\hline Yes & $11(84.6)$ & $8(72.7)$ & \\
\hline No & $2(15.4)$ & $3(27.3)$ & \\
\hline Hormonal therapy, №. (\%) & & & 1.000 \\
\hline Yes & $8(61.5)$ & $7(63.6)$ & \\
\hline No & $5(38.5)$ & $4(36.4)$ & \\
\hline Anti-HER2 therapy, No. (\%) & & & 1.000 \\
\hline Yes & $5(38.5)$ & $5(45.5)$ & \\
\hline No & $8(61.5)$ & $6(54.5)$ & \\
\hline Radiotherapy, No. (\%) & & & - \\
\hline Yes & $13(100.0)$ & $11(100.0)$ & \\
\hline No & $0(0)$ & $0(0)$ & \\
\hline Arm lymphedema, No. (\%) & $1(7.7)$ & $2(18.2)$ & $0.877^{\mathrm{a}}$ \\
\hline Locoregional recurrence, No. (\%) & $0(0)$ & $0(0)$ & - \\
\hline
\end{tabular}

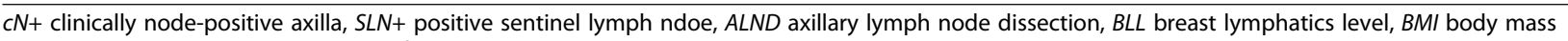
index, HER2 human epidermal growth factor receptor 2

${ }^{a}$ Chi-square test, bnonparametric test 
controlled trial comparing ALND based on BLL and standard ALND revealed a satisfactory outcome. Further randomized controlled trial was needed to confirm its effect.

\section{Conclusion}

With the ALND based on BLL approach, a more focused and less radical axillary dissection to remove the disease can be performed. To determine the precise scope of the axillary dissection, a horizontal line $1.0 \mathrm{~cm}$ away from the superior blue-stained bALN and a vertical line 1.0 $\mathrm{cm}$ away from the medial blue-stained bALN formed an LOQ zone in the axilla, which was defined as BLL II, III, IV. This new classification of the breast lymphatics could minimize the axillary dissection for breast cancer patients with pathological node-positive axilla and the potential to reduce BCRL events.

\section{Supplementary Information}

The online version contains supplementary material available at https://doi. org/10.1186/s12885-021-08024-y.

Additional file 1: Supplement 1. The staged tracing procedure where

$0.1 \mathrm{ml}$ methylene blue is injected into the sentinel nodes

\section{Abbreviations}

SLNB: Sentinel lymph node biopsy; ALND: Axillary lymph node dissection; cN+: clinically node-positive axilla; BCRL: Breast cancer-related lymphedema; SLN: Sentinel lymph node; bALN: axillary lymph node from the breast; BLL: Breast lymphatics level; NCT: Neoadjuvant chemotherapy; ICG: Indocyanine green; MB: Methylene blue; FS: Frozen sectioning; LOQ: Lower outer quadrant; ARM: Axillary reverse mapping;

CARE: Conservative axillary regional excision

\section{Acknowledgements}

The authors thank the studied patients for their willingness to cooperate with our study.

\section{Authors' contributions}

Conceptualization: WG, YQ; Investigation and Data curation: WG, YQ, HJ, HY and ZL; Methodology, Formal analysis and Validation: WG, LY; Writing and editing: YQ, HY and ZL. Resources and Supervision: WG and HJ; All authors have read and approved the manuscript.

\section{Funding}

This study is supported by grants from Improving the Ability of Diagnosis and Treatment of Difficult Diseases (Oncology) Registered Clinical Project of Wuhan University Zhongnan Hospital (ZLYNXM202014). None of the funding bodies had any part in the design of the study, collection, analysis, and interpretation of data, or in writing the manuscript.

\section{Availability of data and materials}

Due to the privacy of patients, the data related to patients cannot be available for public access but can be obtained from the corresponding author on reasonable request approved by the institutional review board of Wuhan University of Zhongnan Hospital. (wugaosong@whu.edu.cn).

\section{Declarations}

\section{Ethics approval and consent to participate}

This research was comprised of human participants and was approved by Medical Ethics Committee of Wuhan University Zhongnan Hospital (ID: 2017047). Written informed consent was obtained from all participants.
Consent for publication

Not applicable.

\section{Competing interests}

The authors declare that they have no competing interests.

Received: 28 August 2020 Accepted: 10 March 2021

Published online: 19 March 2021

\section{References}

1. Giuliano AE, Kirgan DM, Guenther JM, Morton DL. Lymphatic mapping and sentinel lymphadenectomy for breast cancer. Ann Surg. 1994;220(3):391-8, 398-401. https://doi.org/10.1097/00000658-199409000-00015.

2. Togawa K, Ma H, Sullivan-Halley J, Neuhouser ML, Imayama I, Baumgartner KB, Smith AW, Alfano CM, McTiernan A, Ballard-Barbash R, Bernstein L. Risk factors for self-reported arm lymphedema among female breast cancer survivors: a prospective cohort study. Breast Cancer Res. 2014;16(4):414. https://doi.org/10.1186/s13058-014-0414-x

3. Nguyen TT, Hoskin TL, Habermann EB, Cheville AL, Boughey JC. Breast Cancer-related lymphedema risk is related to multidisciplinary treatment and not surgery alone: results from a large cohort study. Ann Surg Oncol. 2017;24(10):2972-80. https://doi.org/10.1245/s10434-017-5960-x.

4. Giuliano AE, Ballman K, McCall L, Beitsch P, Whitworth PW, Blumencranz P Leitch AM, Saha S, Morrow M, Hunt KK. Locoregional recurrence after sentinel lymph node dissection with or without axillary dissection in patients with sentinel lymph node metastases. Ann Surg. 2016;264(3):41320. https://doi.org/10.1097/SLA.0000000000001863.

5. Suami H, Pan W, Mann GB, Taylor Gl. The lymphatic anatomy of the breast and its implications for sentinel lymph node biopsy: a human cadaver study. Ann Surg Oncol. 2008;15(3):863-71. https://doi.org/10.1245/s10434007-9709-9.

6. Giuliano AE, Ballman KV, McCall L, Beitsch PD, Brennan MB, Kelemen PR, Ollila DW, Hansen NM, Whitworth PW, Blumencranz PW, Leitch AM, Saha S, Hunt KK, Morrow M. Effect of axillary dissection vs no axillary dissection on 10-year overall survival among women with invasive breast Cancer and sentinel node metastasis: the ACOSOG Z0011 (Alliance) randomized clinical trial. JAMA. 2017;318(10):918-26. https://doi.org/10.1001/jama.2017.11470.

7. Benson JR, Della RG. Management of the axilla in women with breast cancer. Lancet Oncol. 2007;8(4):331-48. https://doi.org/10.1016/S1470-204 5(07)70103-1.

8. BERG JW. The significance of axillary node levels in the study of breast carcinoma. Cancer-Am Cancer Soc. 1955;8(4):776-8.

9. Yamamoto S, Maeda N, Yoshimura K, Oka M. Intraoperative detection of sentinel lymph nodes in breast cancer patients using ultrasonographyguided direct indocyanine green dye-marking by real-time virtual sonography constructed with three-dimensional computed tomographylymphography. Breast. 2013;22(5):933-7. https://doi.org/10.1016/j.breast.2 013.05.001.

10. Yuan Q, Wu G, Xiao S, He Y, Wang K, Zhang D. Surgical Management of the Axilla in breast Cancer patients with negative sentinel lymph node: a method to reduce false-negative rate. World J Surg. 2019;43(4):1047-53. https://doi.org/10.1007/s00268-018-4865-6.

11. DiSipio T, Rye S, Newman B, Hayes S. Incidence of unilateral arm lymphoedema after breast cancer: a systematic review and meta-analysis. Lancet Oncol. 2013;14(6):500-15. https://doi.org/10.1016/S1470-2045(13 )70076-7.

12. Ochoa D, Korourian S, Boneti C, Adkins L, Badgwell B, Klimberg VS. Axillary reverse mapping: five-year experience. Surgery. 2014;156(5):1261-8. https:// doi.org/10.1016/j.surg.2014.05.011.

13. Thompson M, Korourian S, Henry-Tillman R, Adkins L, Mumford S, Westbrook KC, Klimberg VS. Axillary reverse mapping (ARM): a new concept to identify and enhance lymphatic preservation. Ann Surg Oncol. 2007;14(6): 1890-5. https://doi.org/10.1245/s10434-007-9412-x.

14. Nos C, Lesieur B, Clough KB, Lecuru F. Blue dye injection in the arm in order to conserve the lymphatic drainage of the arm in breast Cancer patients requiring an axillary dissection. Ann Surg Oncol. 2007;14(9):2490-6. https:// doi.org/10.1245/s10434-007-9450-4.

15. Tummel E, Ochoa D, Korourian S, Betzold R, Adkins L, McCarthy M, Hung S, Kalkwarf K, Gallagher K, Lee JY, Klimberg VS. Does axillary reverse mapping prevent lymphedema after lymphadenectomy? Ann Surg. 2017:265(5):98792. https://doi.org/10.1097/SLA.0000000000001778. 
16. Ochoa DA. Axillary lymphatic evaluation: a solution to a complex problem. Ann Surg Oncol. 2019;26(11):3413-4. https://doi.org/10.1245/s10434-01907570-X.

17. Yuan $Q$, Wu G, Xiao S, Hou J, Ren $Y$, Wang $H$, et al. Identification and preservation of arm lymphatic system in axillary dissection for breast Cancer to reduce arm lymphedema events: a randomized clinical trial. Ann Surg Oncol. 2019;26(11):3446-54. https://doi.org/10.1245/s10434-019-07569-4.

18. Beek MA, Verheuvel NC, Luiten EJ, Klompenhouwer EG, Rutten HJ, Roumen $\mathrm{RM}$, et al. Two decades of axillary management in breast cancer. Br I Surg. 2015;102(13):1658-64. https://doi.org/10.1002/bjs.9955.

19. Livingston EH, Li HC. Breast Cancer surgery: less is more. JAMA. 2017; 318(10):909-11. https://doi.org/10.1001/jama.2017.12890.

20. Poodt I, Spronk P, Vugts G, van Dalen T, Peeters M, Rots ML, et al. Trends on Axillary Surgery in Nondistant Metastatic Breast Cancer Patients Treated Between 2011 and 2015. Ann Surg. 2018;268(6):1084-90. https://doi.org/10.1 097/SLA.00000000000002440.

21. Caudle AS, Yang WT, Krishnamurthy S, Mittendorf EA, Black DM, Gilcrease MZ, Bedrosian I, Hobbs BP, DeSnyder SM, Hwang RF, Adrada BE, Shaitelman SF, Chavez-MacGregor M, Smith BD, Candelaria RP, Babiera GV, Dogan BE, Santiago L, Hunt KK, Kuerer HM. Improved axillary evaluation following Neoadjuvant therapy for patients with node-positive breast Cancer using selective evaluation of clipped nodes: implementation of targeted axillary dissection. J Clin Oncol. 2016;34(10):1072-8. https://doi.org/10.1200/JCO.201 5.64.0094.

22. Goyal A, Duley L, Fakis A. Axillary treatment for patients with early breast cancer and lymph node metastasis: systematic review protocol. World $J$ Surg Oncol. 2013;1 1(1):6. https://doi.org/10.1186/1477-7819-11-6.

23. Martin RCG, Chagpar A, Scoggins CR, Edwards MJ, Hagendoorn L, Stromberg AJ, et al. Clinicopathologic factors associated with false-negative sentinel lymph-node biopsy in breast Cancer. Ann Surg. 2005;241(6):100515. https://doi.org/10.1097/01.sla.0000165200.32722.02.

\section{Publisher's Note}

Springer Nature remains neutral with regard to jurisdictional claims in published maps and institutional affiliations.

Ready to submit your research? Choose BMC and benefit from:

- fast, convenient online submission

- thorough peer review by experienced researchers in your field

- rapid publication on acceptance

- support for research data, including large and complex data types

- gold Open Access which fosters wider collaboration and increased citations

- maximum visibility for your research: over $100 \mathrm{M}$ website views per year

At $\mathrm{BMC}$, research is always in progress.

Learn more biomedcentral.com/submissions 